\title{
MiR-145-5p suppresses the proliferation, migration and invasion of gastric cancer epithelial cells via the ANGPT2/NOD_LIKE_ RECEPTOR axis
}

\author{
Kai Zhou ${ }^{1 \dagger}$, Binbin Song ${ }^{2+}$, Ming Wei ${ }^{1}$, Jubo Fang ${ }^{1}$ and Yufen $\mathrm{Xu}^{2 *}$
}

\begin{abstract}
Objective: This study aimed to investigate the relationship among miR-145-5p, ANGPT2 and the NOD_LIKE_RECEPTOR pathway, thereby revealing the molecular mechanism of these three factors underlying the proliferation, migration and invasion of gastric cancer (GC) epithelial cells.

Methods: qRT-PCR was carried out to detect the expression of miR-145-5p and ANGPT2 mRNA. Western blot was performed to test the protein levels of ANGPT2 as well as NOD1, NOD2 and NF-KB in the NOD_LIKE_RECEPTOR pathway. The targeting relationship between miR-145-5p and ANGPT2 was verified via a dual-luciferase reporter gene assay. The proliferation, migration and invasion of GC cells were detected through MTT and Transwell assays, respectively.

Results: The expression of miR-145-5p was significantly down-regulated in GC cells, while that of ANGPT2 was notably up-regulated. MiR-145-5p directly bound with the 3'-UTR of ANGPT2 mRNA, thereby targeting ANGPT2 after transcription. Overexpression of miR-145-5p inhibited the proliferation, migration and invasion of GC cells by suppressing ANGPT2. Moreover, low expression of ANGPT2 affected the protein levels of NOD1, NOD2 and NF-KB in the NOD_LIKE_RECEPTOR pathway, thus weakening the abilities of cell proliferation, migration and invasion.

Conclusions: MiR-145-5p plays an important role in GC epithelial cells, and it can affect cell proliferation, migration and invasion of GC cells by targeting ANGPT2 and regulating the NOD_LIKE_RECEPTOR pathway. Overall, our study further elucidates the molecular mechanism underlying the malignant progression of GC.
\end{abstract}

Keywords: miR-145-5p, ANGPT2, NOD_LIKE_RECEPTOR, Gastric cancer, Proliferation, Migration and invasion

\section{Background}

Gastric cancer (GC) is one of the four most common malignancies worldwide with a relatively high rate of morbidity and mortality [1]. Because of unobvious

\footnotetext{
*Correspondence: xyf_8829@163.com

†Kai Zhou and Binbin Song contributed equally to this work

${ }^{2}$ Department of Oncology, The First Hospital of Jiaxing, Affiliated Hospital

of Jiaxing University, 1882\# Zhonghuan South Road, Jiaxing 314000, China
}

Full list of author information is available at the end of the article symptoms in the early stage of $\mathrm{GC}$, most patients are diagnosed in the advanced stage, and surgical resection is no more effective, leading to a low cure rate and poor overall prognosis [2]. In addition, the incidence of GC in young people has gradually increased [3]. Although great progress has been achieved in the diagnosis and treatment of GC in recent years, the survival rate of this disease in most countries still remains $25-30 \%$ [4]. Therefore, investigating the molecular mechanism underlying $\mathrm{GC}$ initiation and progression is of great importance for exploring targeted therapeutic approaches towards GC .

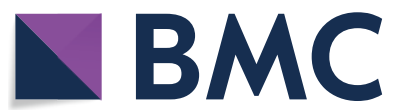

(c) The Author(s) 2020. This article is licensed under a Creative Commons Attribution 4.0 International License, which permits use, sharing, adaptation, distribution and reproduction in any medium or format, as long as you give appropriate credit to the original author(s) and the source, provide a link to the Creative Commons licence, and indicate if changes were made. The images or other third party material in this article are included in the article's Creative Commons licence, unless indicated otherwise in a credit line to the material. If material is not included in the article's Creative Commons licence and your intended use is not permitted by statutory regulation or exceeds the permitted use, you will need to obtain permission directly from the copyright holder. To view a copy of this licence, visit http://creativecommons.org/licenses/by/4.0/. The Creative Commons Public Domain Dedication waiver (http://creativecommons.org/publicdomain/zero/1.0/) applies to the data made available in this article, unless otherwise stated in a credit line to the data. 
MicroRNAs (miRNAs), a type of small endogenous RNA, can negatively regulate gene expression in the post-transcriptional level [5-7], and most of them can pair with the complementary sites within their target mRNAs [8]. Previous studies suggested that miRNAs are involved in the proliferation, migration and invasion of cancer cells $[9,10]$. For instance, miR-137 plays an anti-tumor role in astrocytoma cells through targeting RASGRF1 [10]. In esophageal squamous carcinoma, miR$125 \mathrm{~b}$ targets $B M F$, thereby inhibiting cell proliferation and inducing apoptosis [11]. Furthermore, miR-183-5p and miR-486-5p are found to be significantly correlated with TNM staging in GC patients, while miR-30c-2-3p and miR-133a-3p are associated with the degree of tumor differentiation and lymph node metastasis [12].

Angiogenesis is the formation of new blood vessels from the pre-existing ones. These new blood vessels can be used to form a new vascular network in tumor, thereby promoting a series of cell activities, such as proliferation, migration, invasion and adhesion. Therefore, angiogenesis is crucial during the occurrence and development of various malignant tumors [13-15]. More evidence has revealed that Angiopoietin-2 (ANGPT2, Ang2) can function on angiogenesis and directly stimulate the proliferation of cancer cells [16]. In addition, as an antagonist of ANGPT1, ANGPT2 can suppress the autophosphorylation of Tie2 and break the stabilization of blood vessels. In the presence of vascular endothelial growth factors (VEGFs), vessels that are damaged might be repaired with the occurrence of angiogenesis. In other words, $A N G P T 2$ can cooperate with VEGFs to induce angiogenesis, whereas ANGPT1 can offset VEGFs-induced angiogenesis in vivo $[17,18]$. As the level of $A N G P T 2$ is up-regulated in plasma in cancer patients and associated with poor prognosis [19-21], ANGPT2 can be used as a biomarker for cancer treatment.

NOD-like receptor (NLR) is a type of pattern recognition receptor (PRR) that plays a vital role in immunoregulation [22]. It has been reported that NLR-mediated inflammation is associated with several tumor activities, such as tumorigenesis, progression, metastasis and survival [23]. Additionally, NLR signaling pathways have a significant role in numerous human diseases, including bacterial infections, autoimmune and inflammatory disorders, and cancers [22].

Studies have suggested that miRNAs can be involved in the regulation of angiogenesis via affecting the angiogenesis factors in endothelial cells [24]. For example, miR-126 [25] and miR-210 [26] can facilitate angiogenesis, while miR-221 and miR-222 [27] play a negative role. In the present study, we investigated the regulatory relationship between miR-145-5p and ANGPT2, thereby revealing the effect of these two factors on the cell proliferation, migration and invasion of GC.

\section{Materials and methods Cell culture}

Human GC cell lines BGC-823 (BNCC100086), MGC803 (BNCC340396), SGC-7901 (BNCC100114), HGC-27 (BNCC100097), AGS (BNCC102154) and normal gastric cell line GES-1 (BNCC342032) were purchased from BeNa Culture Collection (Beijing, China). All cells were cultured in the RPMI-1640 medium (Gibco, Thermo Fisher Scientific, Inc., Waltham, MA, USA) supplemented with $10 \%$ fetal bovine serum (FBS; Hyclone; GE Healthcare Life Sciences, Logan, UT, USA) as well as 100 $\mathrm{U} / \mathrm{mL}$ streptomycin (Gibco, Thermo Fisher Scientific, Inc.) and $100 \mathrm{U} / \mathrm{mL}$ penicillin (Gibco, Thermo Fisher Scientific, Inc.) under the environment of $5 \% \mathrm{CO}_{2}$ at $37{ }^{\circ} \mathrm{C}$. The medium was replaced on a regular basis.

\section{Cell transfection}

MiR-145-5p mimics and NC mimics were purchased from Ribo Bio (Guangzhou, People's Republic of China). Si-ANGPT2 and si-NC were ordered from GenePharma (Shanghai, China). Lentiviral expression vector pLVX-IRES-neo (Clontech, USA) was used to construct ANGPT2 overexpression vector (oe-ANGPT2), which was then used to infect GC cells with the empty pLVXIRES-neo vector as control. For preparation, all cells were cultured in complete medium for at least $24 \mathrm{~h}$, and then rinsed by phosphate buffered saline (PBS, pH7.4). Transfection was carried out using Lipofectamine2000 (Thermo Fisher Scientific, Inc.), and cells were cultured in corresponding mediums with $5 \% \mathrm{CO}_{2}$ at $37{ }^{\circ} \mathrm{C}$. Blank group was taken as the control only with the transfection reagent.

\section{qRT-PCR}

Total RNA of GC cells was extracted by Trizol kits (Thermo Fisher Scientific), and then taken for reverse transcription using the cDNA synthesis kits (Thermo Fisher Scientific) for the access of cDNA.

qRT-PCR was carried out under the thermal cycling conditions using the miScript SYBR Green PCR Kits (Qiagen, Hilden, Germany): pre-degeneration at $95{ }^{\circ} \mathrm{C}$ for $10 \mathrm{~min}, 40$ cycles of $95^{\circ} \mathrm{C}$ for $15 \mathrm{~s}, 60{ }^{\circ} \mathrm{C}$ for $30 \mathrm{~s}$ followed by $72{ }^{\circ} \mathrm{C}$ for $30 \mathrm{~s}$. U6 and GAPDH were taken as the internal references for normalization of the expression levels of miR-145-5p and ANGPT2 mRNA, respectively. Primers used were all from GeneCopoeia (Guangzhou, China), and sequences are listed in Table 1.

Relative expression levels of the target mRNA and miRNA in the control and experimental groups were 
Table 1 Primer sequence

\begin{tabular}{ll}
\hline Gene & Primer sequence \\
\hline miR-145-5p [28] & F: 5'-GTCCAGTTTTCCCAGGAATC-3' \\
& R: 5'-AGAACAGTATTTCCAGGAAT-3' \\
U6 & F: 5'-CTCGCTTCGGCAGCACA-3' \\
& R: 5'-AACGCTTCACGAATTTGCGT-3' \\
ANGPT2 [29] & F: 5'-AGATTTTGGACCAGACCAGTGA-3' \\
& R: 5'-GGATGATGTGCTTGTCTTCCAT-3' \\
GAPDH & F: 5'-GGAGCGAGATCCCTCCAAAAT-3' \\
& R: 5'-GGCTGTTGTCATACTTCTCATGG-3' \\
\hline
\end{tabular}

compared with the $2^{-\Delta \Delta C t}$ value. The experiment was repeated three times.

\section{Western blot}

After $48 \mathrm{~h}$ of incubation, cells were washed by cold PBS three times and lysed on ice with whole cell lysate for 10 min. Proteins contained were quantitated by BCA protein assay kit (Thermo Fisher Scientific, Waltham, MA, USA). A measure of $30 \mu \mathrm{g}$ of total proteins were then separated by polyacrylamide gel electrophoresis (PAGE) at a constant voltage of $80 \mathrm{~V}$ for $35 \mathrm{~min}$ followed by $120 \mathrm{~V}$ for $45 \mathrm{~min}$, and sequentially transferred onto the polyvinylidene fluoride (PVDF) membranes (Amersham, USA) after electrophoresis. The membranes were blocked with $5 \%$ skim milk at room temperature for $1 \mathrm{~h}$. Afterwards, the membranes were incubated overnight at $4{ }^{\circ} \mathrm{C}$ with primary rabbit polyclonal antibodies containing ANGPT2 (ab155106, 1:1000, abcam, Cambridge, UK), NOD1 (ab217798, 1:1000, abcam), NOD2 (ab36836, 1:1000, abcam), NF-kB (ab194729, 1:1000, abcam) and GAPDH (ab9485, 1:2500, abcam). Then, the membranes were incubated with secondary antibody, horse radish peroxidase-labeled goat anti-rabbit IgG H\&L (HRP) (ab6721, 1:2000, abcam) for $1 \mathrm{~h}$. Protein bands were visualized under the optical luminescence instrument (GE, USA), and were quantified by gray scale scanning using the Image Pro Plus 6.0 software (Media Cybernetics, USA).

\section{MTT assay}

MTT method (Sigma, Shanghai) was applied for proliferation detection of GC cells. Cells were seeded into 96-well plates at a density of $2 \times 10^{3}$ cells/well. After $24 \mathrm{~h}, 48$ and $72 \mathrm{~h}$, respectively, $20 \mu \mathrm{L}$ of MTT $(0.5 \mathrm{mg} /$ $\mathrm{mL}$; Sigma-Aldrich, St. Louis, MO, USA) was added into each well for incubation at $37{ }^{\circ} \mathrm{C}$ for $4 \mathrm{~h}$. The precipitate was solubilized in $150 \mu \mathrm{L}$ of RPMI-1640. The OD values at $570 \mathrm{~nm}$ were recorded. The experiment was repeated three times.

\section{Transwell}

\section{Migration assay}

Cells in the logarithmic phase were firstly starved for $24 \mathrm{~h}$. On the following day, cells were digested, centrifuged and resuspended to a concentration of $2 \times 10^{5}$ cells $/ \mathrm{mL}$. $0.2 \mathrm{~mL}$ of cell suspension was added into the top chambers, and $700 \mu \mathrm{L}$ pre-cooled RPMI-1640 medium complemented with $10 \%$ FBS was added into the bottom chambers. After incubation for $24 \mathrm{~h}$ at $37{ }^{\circ} \mathrm{C}$ with $5 \% \mathrm{CO}_{2}$, the non-migrated cells in the top chambers were wiped off with a cotton swab, and the migrated cells were fixed with methanol for $30 \mathrm{~min}$ and stained with $0.1 \%$ crystal violet for $20 \mathrm{~min}$. Then, cells were rinsed, dried and observed under an inverted microscope. Five fields of view were randomly selected for cell count.

\section{Invasion assay}

Transwell invasion assays were performed using 24-well Transwell chambers ( $8 \mu \mathrm{m}$ in aperture, BD Biosciences). GC cells $\left(2 \times 10^{4}\right)$ were added into the top chambers coated by Matrigel matrix (Corning, NY), and RPMI1640 medium containing 10\% FBS was added to the bottom chambers. After incubation at $37{ }^{\circ} \mathrm{C}$ for $24 \mathrm{~h}$, cotton swabs were used to wipe off the cells still in the top chambers, and the invaded cells were stained with crystal violet. Cells were counted in 5 randomly selected fields.

\section{Dual-luciferase reporter assay}

GC cells were seeded into 24-well plates in a density of $6 \times 10^{5}$ cells/well for $24 \mathrm{~h}$ of incubation. Wild type (WT) and mutant (MUT) ANGPT2 3'-UTR were respectively cloned into pmiRGLO (Promega, Madison, WI, USA) vectors for construction of luciferase reporter vectors WT- ANGPT2 and MUT-ANGPT2. The two vectors were respectively co-transfected with miR-145-5p mimics/NC mimics into GC cells, with the Renilla luciferase expression vector pRL-TK (TaKaRa, Dalian, China) as the internal reference. After transfection, cells were cultured in RPMI-1640 medium containing 10\% FBS for 48 h. Dual-luciferase reporter gene assay system (Promega, Madison, WI, USA) was used for luciferase activity examination. The experiment was repeated three times.

\section{Statistical analysis}

All data were processed by SPSS 21.0 statistical software. Measurement data were presented in the form of mean \pm standard deviation (SD). Comparison between two groups was performed by $t$ test, and comparison among multiple groups was analyzed by one-way ANOVA. $p<0.05$ was considered statistically significant. 


\section{Results}

MiR-145-5p is poorly expressed in GC, while ANGPT2 is highly expressed

Expression profiles of STAD-associated miRNAs were accessed from TCGA database, including 45 normal samples and 444 GC tissue samples. "edgeR" package of $\mathrm{R}$ language was used to perform differential analysis with the normal samples as control. $|\log F C|>2$ and adj. $\mathrm{p}$ value $<0.01$ were set as the threshold. As shown in Fig. 1a and 46 differentially expressed miRNAs were obtained, among which miR-145 was found to be stably expressed in both the normal and cancer samples, but the expression showed a remarkable difference and the FDR value was statistically significant (Fig. 1b). According to the starBase database (http://starbase.sysu.edu.cn/ index.php), miR-145-5p is considered the most probable mature form of miR-145.

It has been reported that miR-145-5p is closely related to tumorigenesis and progression [30, 31]. In order to investigate the downstream regulatory mechanism of miR-145-5p, we firstly screened differentially expressed mRNAs in TCGA-STAD dataset via differential analysis (Fig. 1c). Afterwards, TargetScan (http://www.targetscan .org/vert_71/), miRDB (http://www.mirdb.org/micro DB/policy.html) and miRTarBase (http://mirtarbase .mbc.nctu.edu.tw/php/index.php) three databases were used to predict potential target genes of miR-145-5p. Venn diagram in Fig. 1d was drawn to find the overlapping mRNAs between the target genes predicted and the differentially expressed mRNAs obtained before. Eventually, 3 potential target genes of miR-145-5p were achieved, among which ANGPT2 was significantly upregulated in tumor samples (Fig. 1e). Moreover, 489 samples in TCGA-STAD dataset were grouped into high and low expression groups with the median expression of $A N G P T 2$ as the critical value. Further survival analysis revealed that $A N G P T 2$ expression showed a remarkable effect on prognosis of GC patients, and patients with high expression of $A N G P T 2$ had poor prognosis (Fig. 1f).

To investigate the role of miR-145-5p and ANGPT2 in $\mathrm{GC}$ progression, expression levels of the two genes were detected in 5 GC cell lines BGC-823, MGC-803, SGC-7901, HGC-27, AGS and one normal gastric cell line GES-1. As shown in Fig. 1g, h, results of qRT-PCR suggested that the expression of miR-145-5p was significantly down-regulated in 5 GC cell lines by comparison with that in normal cell line GES-1, while ANGPT2 had the opposite effect. For preciseness, BGC-823 and AGS with most significant differential expression of miR145-5p or ANGPT2 were selected for subsequent experiments. Western blot was performed to assess the protein levels of $A N G P T 2$ in these two cell lines (Fig. 1i), and the result obtained was consistent with that by qRT-PCR.

\section{MiR-145-5p inhibits the proliferation, migration and invasion of GC cells}

NC mimic and miR-145-5p mimic were transfected into GC cell lines BGC-823 and AGS, respectively. Results of qRT-PCR (Fig. 2a) showed that the expression of miR145-5p was significantly up-regulated in the miR-145-5p mimic group. MTT assay suggested that cells transfected with miR-145-5p mimic had much lower viability at 48 and $72 \mathrm{~h}$ (Fig. 2b, c). In addition, Transwell assays revealed that overexpression of miR-145-5p played an inhibitory role in cell migration (Fig. 2d) and invasion (Fig. 2e). All these data elucidated that miR-145-5p overexpression could inhibit the proliferation, migration and invasion of GC cells and in turn functioned in tumorigenesis and progression.

\section{MiR-145-5p targets ANGPT2}

As described by bioinformatics analysis, the expression of miR-145-5p was found to be down-regulated in GC cells, while ANGPT2 was up-regulated. In order to verify the regulatory relationship between miR-145-5p and $A N G P T 2$, qRT-PCR and western blot were carried out. As shown in Fig. 3a, b, the mRNA and protein levels of ANGPT2 in the miR-145-5p mimic group were both remarkably decreased by comparison with those in the NC mimic group. Besides, TargetScan website was applied and it was found that there were potential targeted binding sites of miR-145-5p on ANGPT2 3'-UTR region (Fig. 3c). For further verification, luciferase reporter vectors WT-ANGPT2 and MUT-ANGPT2 were constructed (Fig. 3d). As shown in Fig. 3e, in the presence of miR-145-5p overexpression, luciferase activity in the cells transfected with WT-ANGPT2 presented a downward trend, while that with MUT-ANGPT2 showed no

(See figure on next page.)

Fig. 1 MiR-145-5p is poorly expressed in GC, while ANGPT2 is highly expressed. a Volcano plot shows the 46 differentially expressed miRNAs in TCGA-STAD dataset; $\mathbf{b}$ Differential expression of miR-145-5p in normal and tumor groups in TCGA-STAD samples; $\mathbf{c}$ Volcano plot shows the 2760 differentially expressed mRNAs in TCGA-STAD dataset; $\mathbf{d}$ Venn diagram is plotted to find the overlapping mRNAs from the differentially expressed mRNAs in TCGA-STAD dataset and the target genes of miR-145-5p predicted in three bioinformatics databases; e Differential expression of ANGPT2 in normal and tumor groups in TCGA-STAD samples; $\mathbf{f}$ Overall survival curves of patients in high (red) and low (blue) ANGPT2 expression groups; $\mathbf{g}$, $\mathbf{h}$ The expression of miR-145-5p and ANGPT2 mRNA in 5 GC cell lines BGC-823, MGC-803, SGC-7901, HGC-27, AGS and one normal gastric cell line GES-1 is assessed by qRT-PCR; $\mathbf{i}$ The protein expression of ANGPT2 in BGC-823 and AGS cell lines; ${ }^{*} p<0.05$ 

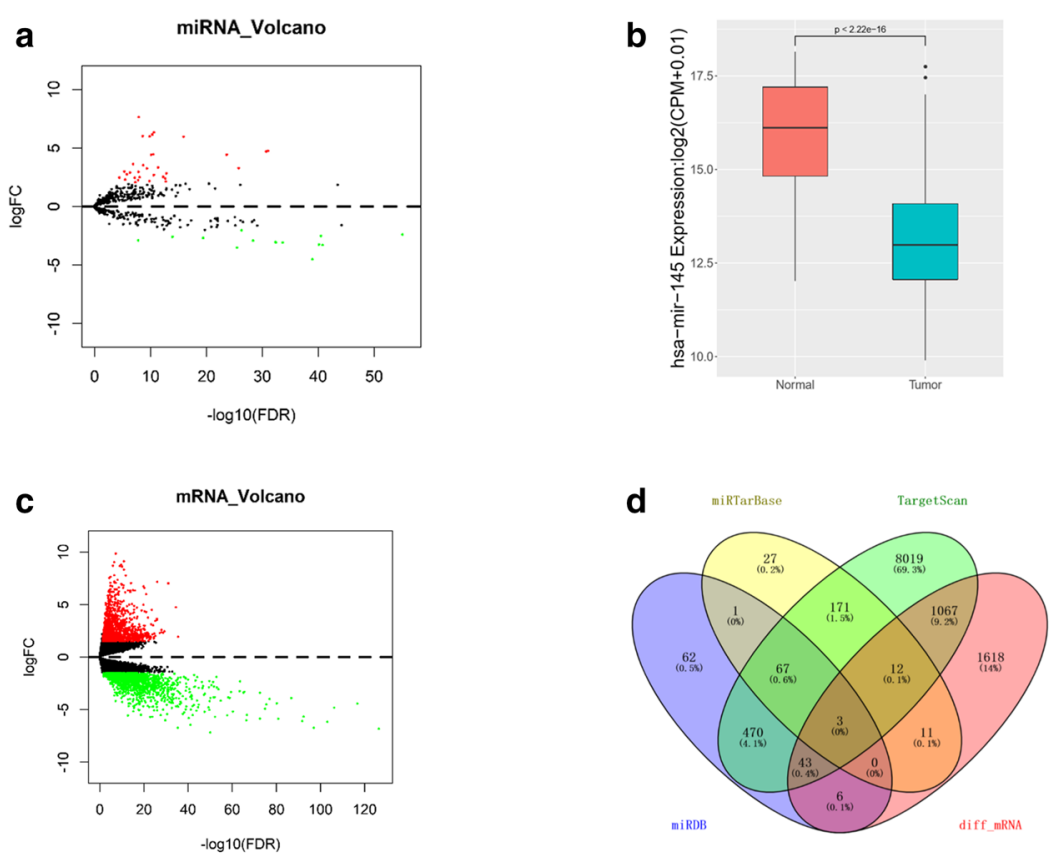

e

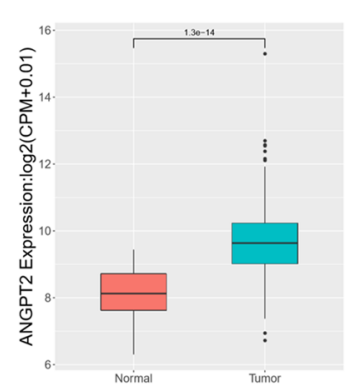

f
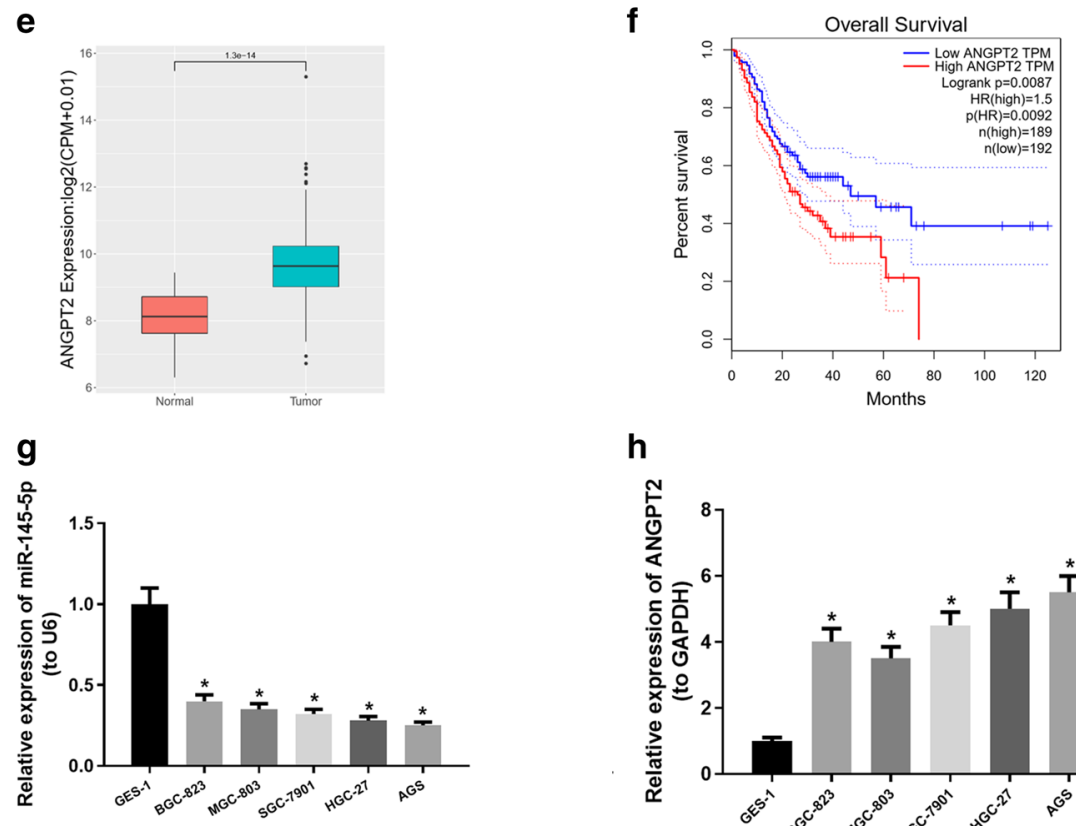

h
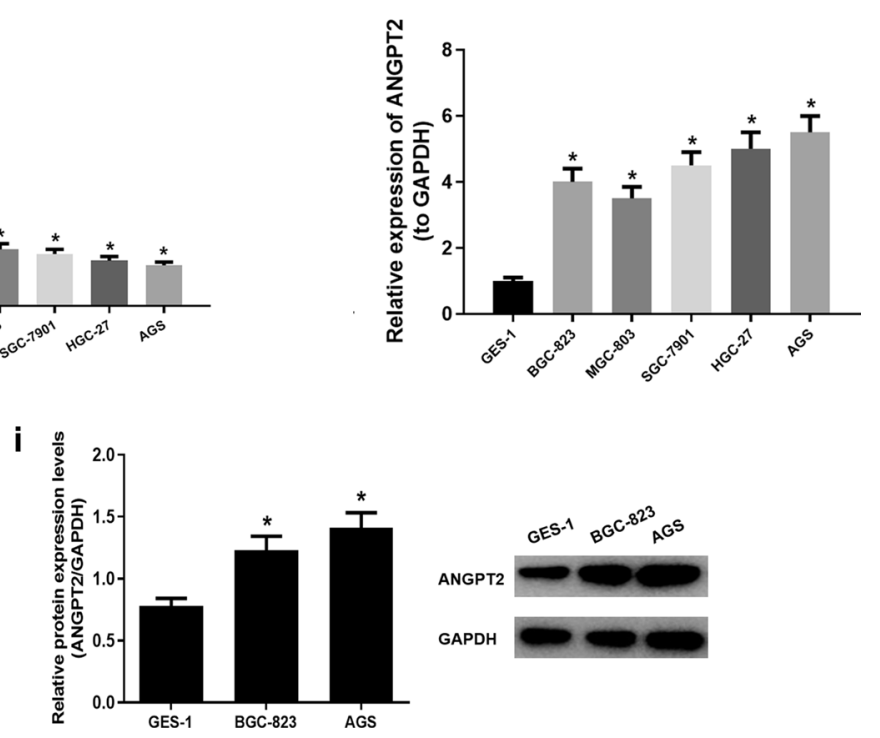


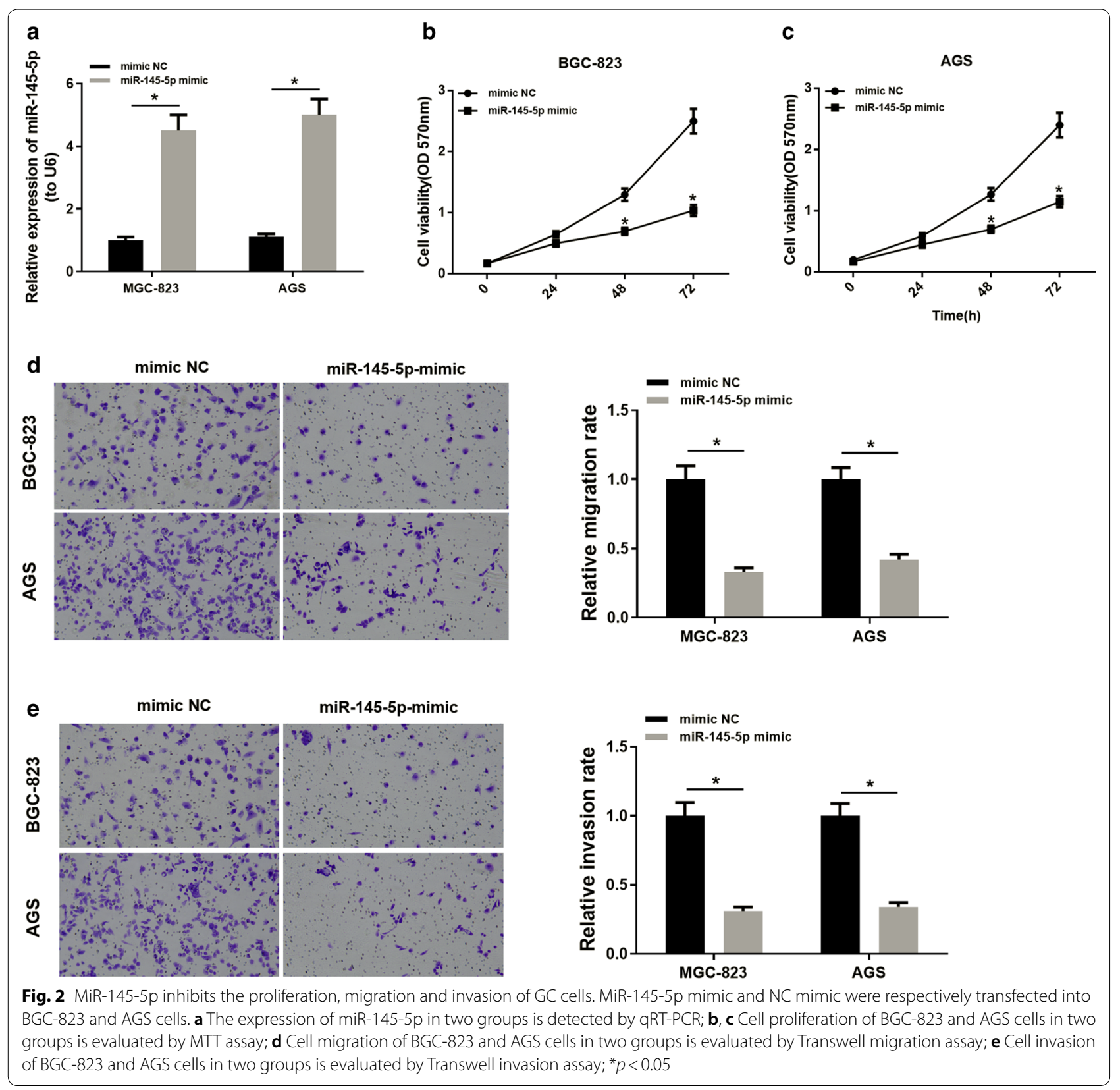

significant difference. All above findings indicated that miR-145-5p could directly target to ANGPT2.

\section{ANGPT2 inhibits the proliferation, migration and invasion of GC cells by regulating the NOD_LIKE_RECEPTOR pathway}

GSEA enrichment analysis was conducted and the result shown in Fig. 4a suggested that ANGPT2 was mostly enriched in the NOD_LIKE_RECEPTOR pathway and NOD1, NOD2 and NF-kB are the key proteins involved in. Therefore, these three key proteins could be used to verify the regulatory effect of $A N G P T 2$ on the NOD_LIKE_RECEPTOR pathway. Results of qRTPCR in Fig. 4b showed that the expression of ANGPT2 was significantly decreased in the si-ANGPT2 group. Meanwhile, western blot indicated that the protein levels of NOD1, NOD2 and NF- $\mathrm{kB}$ were correspondingly decreased in the cells with low ANGPT2 expression (Fig. 4c). Findings above suggested that ANGPT2 could affect the NOD_LIKE_RECEPTOR pathway. In addition, MTT and Transwell assays were performed to verify the effect of ANGPT2 on cancer cell proliferation, migration 


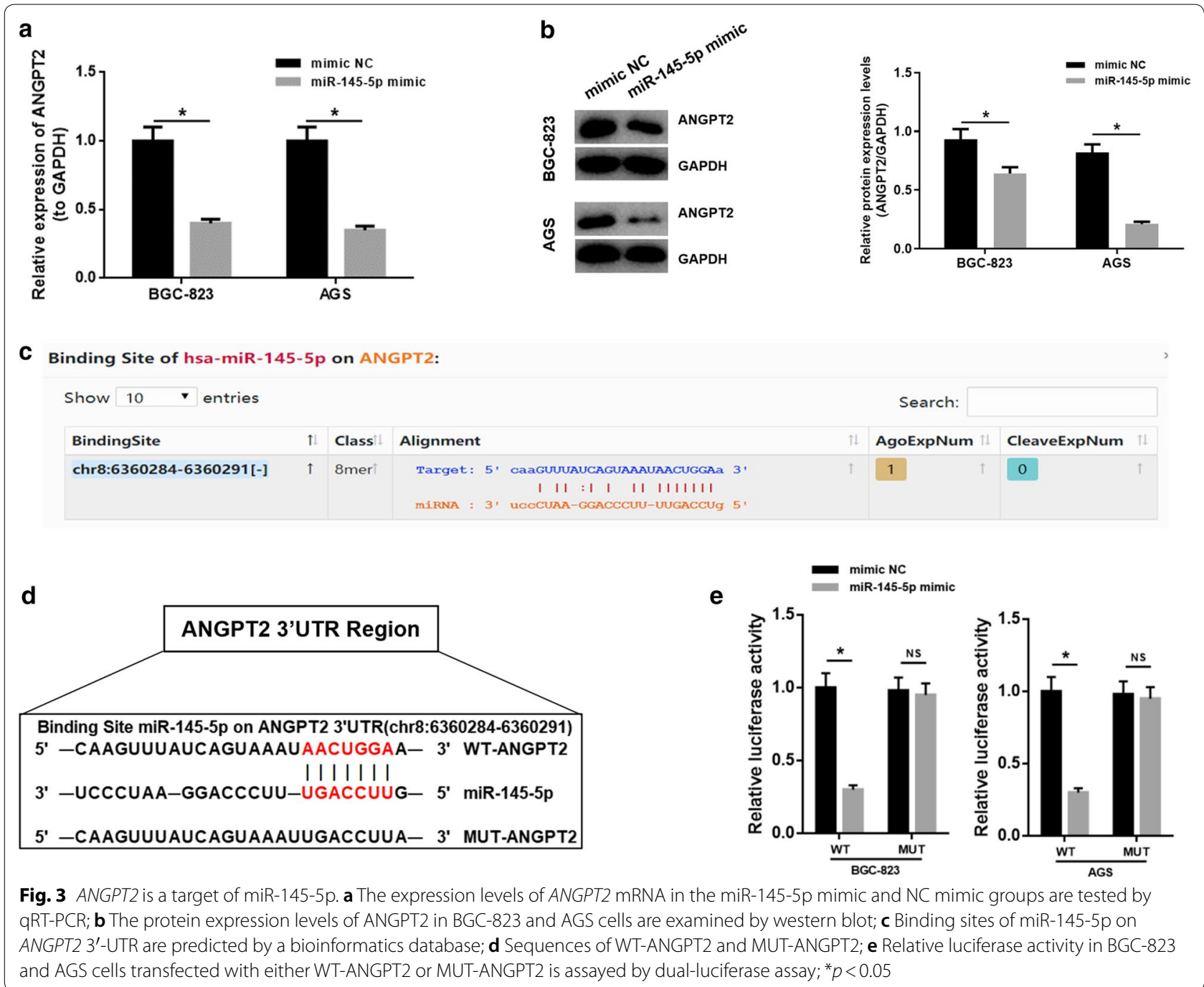

and invasion. As shown in Fig. 4d, cancer cell proliferation was significantly inhibited in the si-ANGPT2 group. Similarly, the migration and invasion of BGC-823 and AGS cells were suppressed as well when ANGPT2 expression was decreased (Fig. 4e, f). Overall, our study confirmed that low expression of ANGPT2 could reduce the proliferation, migration and invasion of GC cells, and suppress its downstream NOD_LIKE_RECEPTOR pathway.

MiR-145-5p suppresses the ANGPT2/NOD_LIKE_RECEPTOR axis to affect $\mathrm{GC}$ progression

In previous experiments, $A N G P T 2$ had been verified to function on the proliferation, migration and invasion of GC cells via the NOD_LIKE_RECEPTOR pathway. As ANGPT2 had been confirmed as a target of miR-145-5p, western blot was firstly performed to investigate the regulatory relationship between miR-145-5p and the
ANGPT2/NOD_LIKE_RECEPTOR axis. The results showed that the expression of ANGPT2 was suppressed in the miR-145-5p mimic group with the blank group as control. In addition, in the cells with overexpression of miR-145-5p and ANGPT2, ANGPT2 expression was significantly lower than that in the oe-ANGPT2 group, which might be attributed to the decrease of ANGPT2 synthesis rate caused by the targeting effect of miR145-5p. Meanwhile, protein levels of NOD1, NOD2 and NF- $\mathrm{BB}$ in the NOD_LIKE_RECEPTOR pathway were detected and found to be relatively high in the groups with high expression of $A N G P T 2$. When miR-145-5p was over-expressed, the protein levels of these proteins were down-regulated to approach to the normal (Fig. 5a).

Furthermore, according to the MTT assay, tumor cells with higher protein levels of NOD1, NOD2 and NF- $\mathrm{KB}$ had a relatively high growth rate (Fig. $5 \mathrm{~b}$ ). Transwell migration and invasion assays revealed that compared 


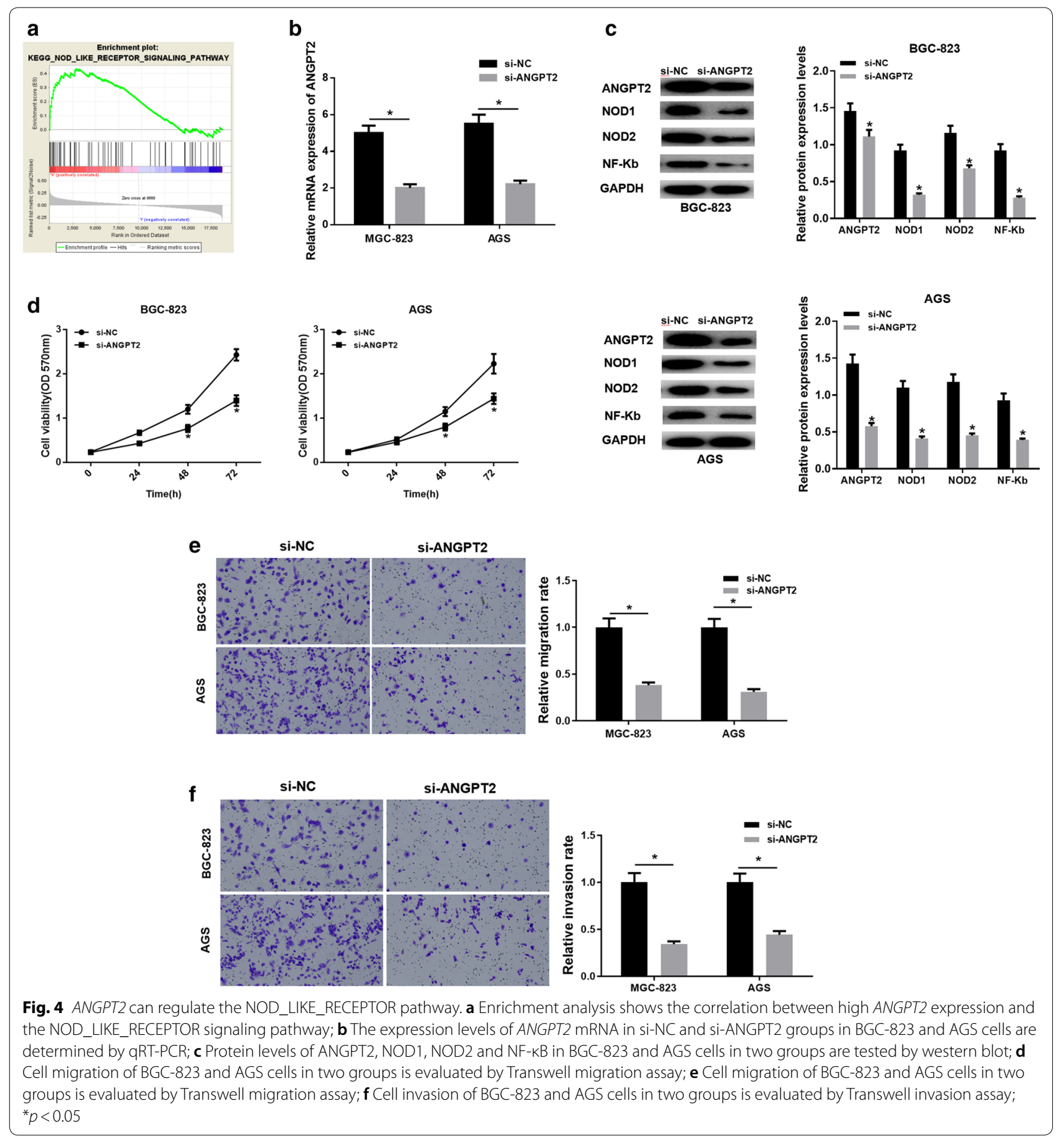

with the blank group, cell migration and invasion were remarkably inhibited in the miR-145-5p mimic group but significantly enhanced in the oe-ANGPT2 group, as shown in Fig. 5c, d. Moreover, in the oeANGPT2 + miR-145-5p mimic group, cell migration and invasion were significantly decreased than those in the oe-ANGPT2 group, which was in agreement with the results obtained above. In sum, miR-145-5p was considered to function on the proliferation, migration and invasion of GC cells via the ANGPT2/NOD_LIKE_ RECEPTOR axis. 


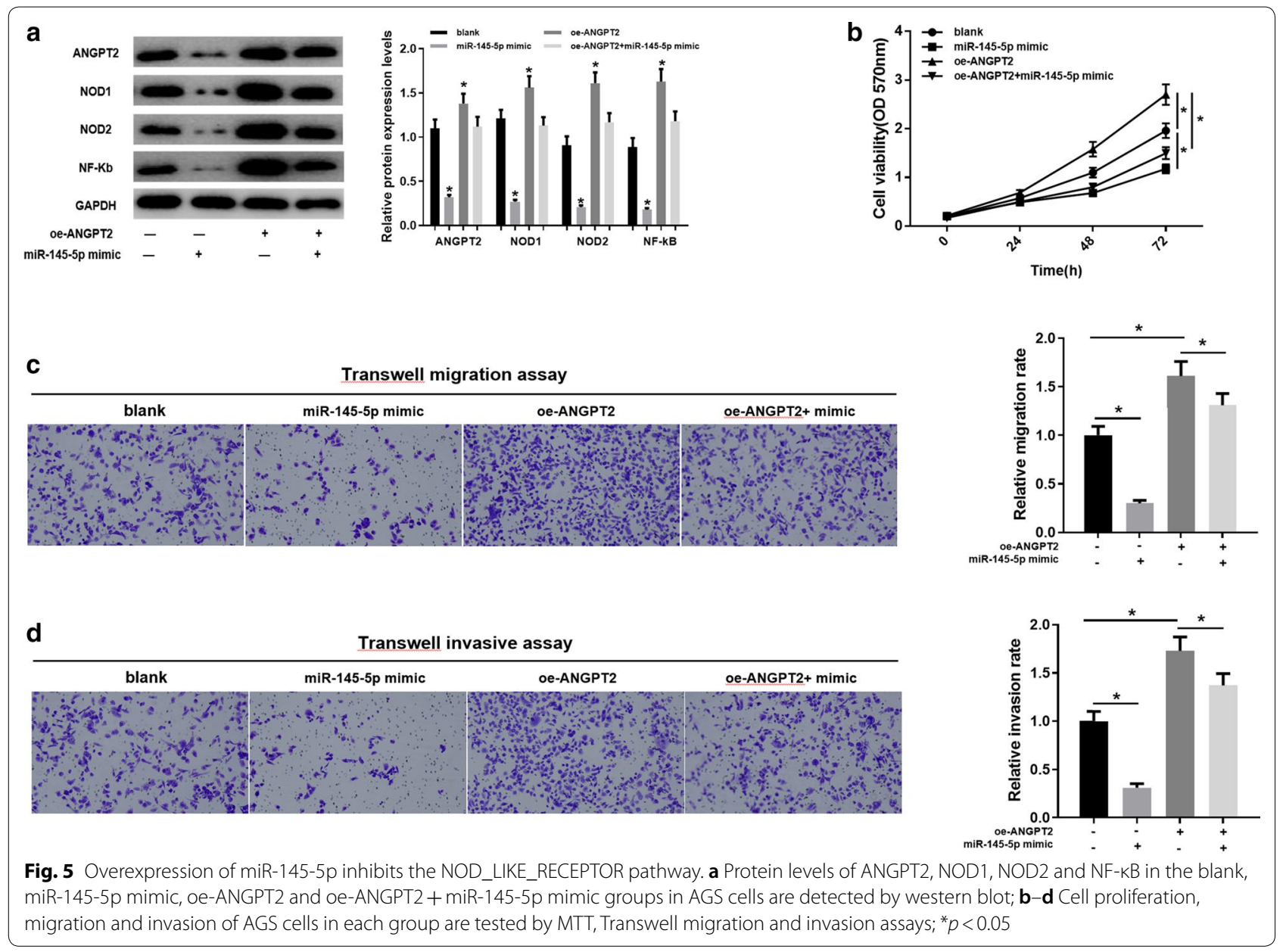

\section{Discussion}

MiRNAs are common in eukaryotes and have been verified to be involved in the regulation of about one third human genes via genome analysis. Each miRNA has hundreds of target genes and participates in the translation or degradation of mRNA in the way of base pairing $[32,33]$. Calin et al. found [34] that among 186 miRNAs, 98 miRNAs (52.5\%) locate at the cancer-related genome regions or fragile sites, and Northern blot results showed that miRNAs in the deleted regions are found to be down-regulated in cancer samples. Many reports have verified that miRNAs can serve as an oncogene or a tumor suppressor gene in cancers, and most of them play an important role in the occurrence and development of GC. For instance, miR-378 inhibits the epithelial-mesenchymal transition (EMT) process, suppresses cell migration and invasion of GC by targeting BMP2 [35]. MiR-107 functions on cell growth and metastasis of GC via the FAT4/PI3K_AKT axis [36]. MiR-145-5p plays a part in various cancers by pairing with its target genes, such as clear cell renal cell carcinoma [37], esophageal squamous carcinoma [38], lung cancer [39] and bladder carcinoma [40]. In GC, miR-145-5p targets $N$-cadherin and ZEB2 to inhibit EMT, thereby inhibiting cell invasion [41]. Furthermore, studies have found that miR-145-5p is down-regulated in undifferentiated GC cells, and directly targets $K L F 5$, thus affecting cell differentiation [42]. In the present study, we confirmed that miR-145-5p was down-regulated in GC cells and significantly associated with cell proliferation, migration and invasion. Therefore, it's of great importance to further elucidate the molecular mechanism of miR-145-5p underlying the tumorigenesis and development of GC.

As previously described, angiogenesis is crucial in the occurrence and development of various malignancies. Thus, molecular treatment methods like the application of the inhibitors of angiogenesis are increasingly important [43]. In most solid tumors, the formation and expansion of blood vessels are tightly correlated with the activity of VEGFs. ANGPT2, a factor suppressed by VEGFs, has been studied on its activity in clinical tumor models, and its up-regulation has been considered as 
one of the mechanisms of acquired drug-resistance during anti-VEGFs treatment [44]. In addition, studies have found that the expression level of ANGPT2 in serum might be a potential predictor in lung cancer staging and correlated with prognosis [45]. Meanwhile, a phase III randomized trial of AVAGAST made by Hacker, et al. revealed that $A N G P T 2$ can function in GC by acting as a biomarker [21]. During our differential analysis based on the TCGA-STAD dataset, ANGPT2 was found to be a potential target of miR-145-5p and significantly up-regulated in GC. Moreover, miR-218 has been reported to inhibit the proliferation and invasion of GC cells through regulating ANGPT2 [29]. Lu et al. confirmed that miR145 plays an anti-tumor role in renal cell carcinoma and it targets two oncogenes including ANGPT2 and NEDD9 [31]. However, whether the regulatory relationship between miR-145 and ANGPT2 functions on GC cells has not been reported yet. In our study, we identified that $A N G P T 2$ was remarkably increased in GC cells, and miR-145-5p could target ANGPT2 mRNA to inhibit the expression of $A N G P T 2$, which is consistent with previous studies.

Besides, KEGG enrichment analysis showed that high expression of ANGPT2 was correlated with the NOD_LIKE_RECEPTOR pathway. Activated NOD1 and NOD2 receptors can be involved in the immunoreaction through the activation of NF-kB, JNK, p38, MAPK and ERK signaling pathways in the way of binding with related signal proteins like serine-threonine protein kinase RIP2, thereby promoting the secretion of various factors, such as IL-6, IL-8, IL- $1 \beta$ and TNF- $\alpha$ [46]. Previous studies suggested that estrogen receptor $\alpha$ regulates the $\mathrm{Wnt} / \beta$ catenin signaling pathway via NOD-like receptors [47]. In our study, results of western blot showed that in GC cells, the protein levels of NOD1, NOD2 and NF- $\mathrm{kB}$ were remarkably increased but significantly decreased after silencing ANGPT2. Thus, we speculated that ANGPT2 could participate in angiogenesis and the proliferation and migration of cancer cells probably through the NOD_LIKE_RECEPTOR signaling pathway.

\section{Conclusions}

In conclusion, our study verifies that miR-145-5p affects the proliferation, migration and invasion of cancer cells via the ANGPT2/NOD_LIKE_RECEPTOR axis, which helps the mechanism research underlying GC cancer progression.

\section{Acknowledgements}

Not applicable.

\section{Authors' contributions}

$\mathrm{KZ}$ and $\mathrm{BS}$ contributed to the study design. MW conducted the literature search. JF acquired the data. YX wrote the article. JF and $Y Z$ revised the article.
$\mathrm{KZ}$ and $\mathrm{BS}$ gave the final approval of the version to be submitted. All authors read and approved the final manuscript.

\section{Funding}

This study was supported by the Funds from The Key Discipline of Jiaxing Oncology Project (2019-zc-11); Science and technology project of Jiaxing (2019AY32030); Jiaxing Key Laboratory of Precision Treatment for Lung Cancer: Key Discipline Established by Zhejiang Province and Jiaxing City Jointly—Pain Medicine(2019-ss-ttyx).

\section{Availability of data and materials}

The data used to support the findings of this study are included within the article. The data and materials in the current study are available from the corresponding author on reasonable request.

\section{Ethics approval and consent to participate}

Not applicable.

\section{Consent for publication}

Not applicable.

\section{Competing interests}

The authors declare no conflicts of interest.

\section{Author details}

${ }_{1}^{1}$ Department of General Surgery, The Dongda Hospital, Shanxian, Heze 274000, China. ${ }^{2}$ Department of Oncology, The First Hospital of Jiaxing, Affiliated Hospital of Jiaxing University, 1882\# Zhonghuan South Road, Jiaxing 314000, China.

Received: 17 May 2020 Revised: 5 August 2020 Accepted: 7 August 2020 Published online: 28 August 2020

\section{References}

1. Carcas LP. Gastric cancer review. J Carcinog. 2014;13:14. https://doi. org/10.4103/1477-3163.146506.

2. GuX, et al. Clinicopathological and prognostic significance of platelet to lymphocyte ratio in patients with gastric cancer. Oncotarget. 2016:7:49878-87. https://doi.org/10.18632/oncotarget.10490.

3. Sun Z, et al. Risk factors associated with splenic hilar lymph node metastasis in patients with advanced gastric cancer in northwest China. Int J Clin Exp Med. 2015;8:21358-64.

4. Karimi P, Islami F, Anandasabapathy S, Freedman ND, Kamangar F. Gastric cancer: descriptive epidemiology, risk factors, screening, and prevention. Cancer Epidemiol Biomarkers Prev. 2014;23:700-13. https://doi. org/10.1158/1055-9965.EPI-13-1057.

5. Ventura A, Jacks T. MicroRNAs and cancer: short RNAs go a long way. Cell. 2009:136:586-91. https://doi.org/10.1016/j.cell.2009.02.005.

6. Shukla GC, Singh J, Barik S, MicroRNAs: processing, maturation, target recognition and regulatory functions. Mol Cell Pharmacol. 2011;3:83-92.

7. Bartel DP. MicroRNAs: target recognition and regulatory functions. Cell. 2009:136:215-33. https://doi.org/10.1016/j.cell.2009.01.002

8. Agarwal V, Bell GW, Nam JW, Bartel DP. Predicting effective microRNA target sites in mammalian mRNAs. Elife. 2015. https://doi.org/10.7554/ eLife.05005.

9. Lu J, et al. MicroRNA expression profiles classify human cancers. Nature. 2005:435:834-8. https://doi.org/10.1038/nature03702.

10. Deng D, et al. miR-137 acts as a tumor suppressor in astrocytoma by targeting RASGRF1. Tumour Biol. 2016;37:3331-40. https://doi.org/10.1007/ s13277-015-4110-y.

11. Fan YX, et al. MicroRNA-125b inhibits cell proliferation and induces cell apoptosis in esophageal squamous cell carcinoma by targeting BMF. Oncol Rep. 2018:40:61-72. https://doi.org/10.3892/or.2018.6413.

12. $L i C Y$, et al. Identification and functional characterization of microRNAs reveal a potential role in gastric cancer progression. Clin Transl Oncol. 2017;19:162-72. https://doi.org/10.1007/s12094-016-1516-y.

13. Yancopoulos GD, et al. Vascular-specific growth factors and blood vessel formation. Nature. 2000;407:242-8. https://doi.org/10.1038/35025215. 
14. Chen Z, et al. Gastric tumour-derived ANGPT2 regulation by DARPP-32 promotes angiogenesis. Gut. 2016;65:925-34. https://doi.org/10.1136/ gutjnl-2014-308416.

15. Sajib S, Zahra FT, Lionakis MS, German NA, Mikelis CM. Mechanisms of angiogenesis in microbe-regulated inflammatory and neoplastic conditions. Angiogenesis. 2018;21:1-14. https://doi.org/10.1007/s1045 6-017-9583-4.

16. Mitsuhashi N, et al. Angiopoietins and Tie-2 expression in angiogenesis and proliferation of human hepatocellular carcinoma. Hepatology. 2003;37:1105-13. https://doi.org/10.1053/jhep.2003.50204.

17. Visconti RP, Richardson CD, Sato TN. Orchestration of angiogenesis and arteriovenous contribution by angiopoietins and vascular endothelial growth factor (VEGF). Proc Natl Acad Sci USA. 2002;99:8219-24. https:// doi.org/10.1073/pnas.122109599.

18. Saharinen P, Eklund L, Alitalo K. Therapeutic targeting of the angiopoietin-TIE pathway. Nat Rev Drug Discov. 2017;16:635-61. https://doi. org/10.1038/nrd.2016.278.

19. Llovet JM, et al. Plasma biomarkers as predictors of outcome in patients with advanced hepatocellular carcinoma. Clin Cancer Res. 2012;18:2290300. https://doi.org/10.1158/1078-0432.CCR-11-2175.

20. Maffei R, et al. Angiopoietin-2 plasma dosage predicts time to first treatment and overall survival in chronic lymphocytic leukemia. Blood. 2010;1 16:584-92. https://doi.org/10.1182/blood-2009-11-252494.

21. Hacker UT, et al. Evaluation of Angiopoietin-2 as a biomarker in gastric cancer: results from the randomised phase III AVAGAST trial. Br J Cancer. 2016;114:855-62. https://doi.org/10.1038/bjc.2016.30.

22. Saxena M, Yeretssian G. NOD-like receptors: master regulators of liflammation and cancer. Front Immunol. 2014;5:327. https://doi.org/10.3389/ fimmu.2014.00327.

23. Janowski AM, Kolb R, Zhang W, Sutterwala FS. Beneficial and detrimental roles of NLRs in carcinogenesis. Front Immunol. 2013;4:370. https://doi. org/10.3389/fimmu.2013.00370.

24. Urbich C, Kuehbacher A, Dimmeler S. Role of microRNAs in vascular diseases, inflammation, and angiogenesis. Cardiovasc Res. 2008;79:581-8. https://doi.org/10.1093/cvr/cvn156.

25. Fish JE, Srivastava D. MicroRNAs: opening a new vein in angiogenesis research. Sci Signal. 2009;2:pe1. https://doi.org/10.1126/scisignal.252pe1.

26. Fasanaro $P$, et al. MicroRNA-210 modulates endothelial cell response to hypoxia and inhibits the receptor tyrosine kinase ligand Ephrin-A3. J Biol Chem. 2008;283:15878-83. https://doi.org/10.1074/jbc.M800731200.

27. Poliseno $L$, et al. MicroRNAs modulate the angiogenic properties of HUVECs. Blood. 2006;108:3068-71. https://doi.org/10.1182/blood-200601-012369.

28. Wang B, Dong W, Li X. miR-145-5p acts as a novel tumor suppressor in hepatocellular carcinoma through targeting RAB18. Technol Cancer Res Treat. 2019;18:1533033819850189. https://doi.org/10.1177/1533033819 850189.

29. Tang S, Wang D, Zhang Q, Li L. miR-218 suppresses gastric cancer cell proliferation and invasion via regulation of angiopoietin-2. Exp Ther Med. 2016;12:3837-42. https://doi.org/10.3892/etm.2016.3893.

30. Ozen $M$, et al. Overexpression of miR-145-5p inhibits proliferation of prostate cancer cells and reduces SOX2 expression. Cancer Invest. 2015:33:251-8. https://doi.org/10.3109/07357907.2015.1025407.

31. Lu R, et al. miR-145 functions as tumor suppressor and targets two oncogenes, ANGPT2 and NEDD9, in renal cell carcinoma. J Cancer Res Clin Oncol. 2014;140:387-97. https://doi.org/10.1007/s00432-013-1577-z.

32. Filipowicz W, Bhattacharyya SN, Sonenberg N. Mechanisms of posttranscriptional regulation by microRNAs: are the answers in sight? Nat Rev Genet. 2008;9:102-14. https://doi.org/10.1038/nrg2290.
33. Lewis BP, Burge CB, Bartel DP. Conserved seed pairing, often flanked by adenosines, indicates that thousands of human genes are microRNA targets. Cell. 2005;120:15-20. https://doi.org/10.1016/j.cell.2004.12.035.

34. Calin GA, et al. Human microRNA genes are frequently located at fragile sites and genomic regions involved in cancers. Proc Natl Acad Sci USA. 2004;101:2999-3004. https://doi.org/10.1073/pnas.0307323101.

35. Yang YJ, Luo S, Wang LS. Effects of microRNA-378 on epithelial-mesenchymal transition, migration, invasion and prognosis in gastric carcinoma by targeting BMP2. Eur Rev Med Pharmacol Sci. 2019;23:5176-86. https:// doi.org/10.26355/eurrev_201906_18182.

36. Wang L, Li K, Wang C, Shi X, Yang H. miR-107 regulates growth and metastasis of gastric cancer cells via activation of the PI3K-AKT signaling pathway by down-regulating FAT4. Cancer Med. 2019;8:5264-73. https:// doi.org/10.1002/cam4.2396.

37. Liep J, et al. Cooperative effect of miR-141-3p and miR-145-5p in the regulation of targets in clear cell renal cell carcinoma. PLOS ONE. 2016;11:e0157801. https://doi.org/10.1371/journal.pone.0157801.

38. Mei LL, et al. miR-145-5p suppresses tumor cell migration, invasion and epithelial to mesenchymal transition by regulating the Sp1/NF-kappaB signaling pathway in esophageal squamous cell carcinoma. Int J Mol Sci. 2017. https://doi.org/10.3390/ijms18091833.

39. Jin M, et al. Long noncoding RNA JPX correlates with poor prognosis and tumor progression in non-small cell lung cancer by interacting with miR145-5p and CCND2. Carcinogenesis. 2019. https://doi.org/10.1093/carcin/ bgz125.

40. Sun M, et al. Circular RNA CEP128 promotes bladder cancer progression by regulating Mir-145-5p/Myd88 via MAPK signaling pathway. Int J Cancer. 2019;145:2170-81. https://doi.org/10.1002/ijc.32311.

41. Jiang SB, et al. MicroRNA-145-5p inhibits gastric cancer invasiveness through targeting $\mathrm{N}$-cadherin and ZEB2 to suppress epithelial-mesenchymal transition. Onco Targets Ther. 2016;9:2305-15. https://doi org/10.2147/OTT.S101853.

42. Zhou T, Chen S, Mao X. miR-145-5p affects the differentiation of gastric cancer by targeting KLF5 directly. J Cell Physiol. 2019;234:7634-44. https ://doi.org/10.1002/jcp.27525.

43. Song $Z, W u Y$, Yang J, Yang D, Fang X. Progress in the treatment of advanced gastric cancer. Tumour Biol. 2017;39:1010428317714626. https ://doi.org/10.1177/1010428317714626.

44. Rigamonti N, et al. Role of angiopoietin-2 in adaptive tumor resistance to VEGF signaling blockade. Cell Rep. 2014;8:696-706. https://doi. org/10.1016/j.celrep.2014.06.059.

45. XuY, et al. The role of serum angiopoietin-2 levels in progression and prognosis of lung cancer: a meta-analysis. Medicine. 2017;96:e8063. https ://doi.org/10.1097/MD.0000000000008063.

46. Watanabe T, et al. NOD1 contributes to mouse host defense against Helicobacter pylori via induction of type I IFN and activation of the ISGF3 signaling pathway. J Clin Invest. 2010;120:1645-62. https://doi.org/10.1172/ JCl39481.

47. Zarrei $\mathrm{M}$, et al. A large data resource of genomic copy number variation across neurodevelopmental disorders. NPJ Genom Med. 2019;4:26. https ://doi.org/10.1038/s41525-019-0098-3.

\section{Publisher's Note}

Springer Nature remains neutral with regard to jurisdictional claims in published maps and institutional affiliations. 